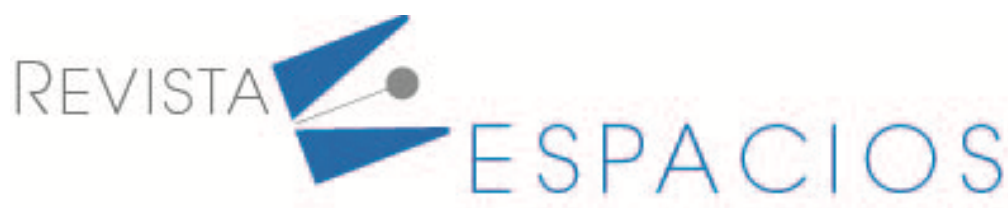

Vol. 41 (50) $2020 \bullet$ Art. 5

Recibido/Received: 24/08/2020 • Aprobado/Approved: 23/11/2020 • Publicado/Published: 30/12/2020

\title{
Microfundamentos de capacidades dinámicas: un estudio de caso sobre la gestión de la manufactura en la fábrica de aceros galvanizados FACAG (Colombia)
}

\section{Microfoundations of dynamic capabilities: a case study on manufacturing management at the Galvanized Steel Factory FACAG}

\author{
CADRAZCO, Wilson G. ${ }^{1}$ \\ ZAPATA, Álvaro ${ }^{2}$ \\ SANTAMARÍA, Álvaro E. ${ }^{3}$
}

\section{Resumen}

La manufactura en la empresa Facag se abordó desde la perspectiva de los microfundamentos de las Capacidades Dinámicas. Se revisó literatura sobre manufactura y su evolución. El estudio cualitativo cruzó información documental con respuestas a las entrevistas, encontrandose evidencia de que la flexibilidad en los procesos de Facag, permiten lograr máximos niveles de eficiencia. Se concluye que los microfundamentos propuestos en la literatura sobre el campo, es decir Detectar, Aprovechar y Reconfigurar, se convierten en solo dos: Detectar y Reconfigurar.

Palabras clave: capacidades dinámicas, manufactura, microfundamentos

\begin{abstract}
Facag company addressed its manufacturing from the microfoundations of Dynamic Capabilities point of view. The literature on manufacturing and its evolution was examined. The qualitative study contrasted documentary information with responses to the interviews, finding proof that reflects the highest levels of efficiency in Facag due to their flexibility processes. In conclusion, the microfoundations that related literature proposes, which are: sensing, seizing, and reconfiguration, become just two: sensing and reconfiguring.

key words: dynamic capabilities, manufacturing, microfoundations
\end{abstract}

\section{Introducción}

Los microfundamentos de las Capacidades Dinámicas, permiten acercarse a estudiar el fenómeno de la obtención de ventaja competitiva por parte de las organizaciones cuando se enfrenta a un entorno incierto y de muchos factores asociados con la existencia de la competencia. Teece, Pisano, \& Shuen (1997), establecieron tres: "Detectar", "Aprovechar", "Reconfigurar", sin embargo, en la presente investigación solo se evidencia la existencia de dos: "Detectar" y "Reconfigurar", al momento de abordar la función de manufactura realizada en

\footnotetext{
${ }^{1}$ Profesor Titular. Facultad de Ciencias Económicas y Administrativas. Universidad de Sucre. Email: wilson.cadrazco@unisucre.edu.co

2 Profesor Titular. Escuela de Negocios. Universidad del Norte. Email: alvaroz@uninorte.edu.co

3 Profesor Asociado. Facultad de Ciencias Económicas y Administrativas. Universidad de Sucre. Email: alvaro.santamaria@unisucre.edu.co
} 
una empresa del sector metalmecánico en Colombia, desde esta perspectiva, surgió esta evidencia que se discute en el presente documento.

\subsection{Revisión de literatura}

El Enfoque de capacidades dinámicas, surge para explicar la sostenibilidad de la ventaja competitiva en ambientes de grandes incertidumbres debidas a los cambios en muchas de las variables del entorno de negocios, así, Teece et al. (1997), las definen como "La capacidad de la empresa para integrar, construir y reconfigurar competencias internas y externas para abordar de manera rápida los cambios en el entorno" (p. 515). Zollo \& Winter (1999), las definen como "Patrón aprendido de la actividad colectiva a través del cual la organización genera y modifica sus rutinas operativas en la búsqueda de una mayor eficacia de manera sistemática” (p.10). Eisenhardt \& Martin (2000) las definen como "las rutinas organizacionales y estratégicas a través de la cual las empresas logran nuevas configuraciones de recursos, debido a que los mercados surgen, chocan, se dividen, evolucionan y mueren" (p. 1107).

Las Capacidades Dinámicas se apoyan en los denominados microfundamentos (Teece, 2003, 2007), establece tres; (1) capacidad para detectar y conformar oportunidades y amenazas, (2) aprovechar oportunidades y (3) mantener la competitividad de la empresa a través de la mejora, la combinación, protección y cuando sea necesario, la reconfiguración de activos intangibles y tangibles. Wang \& Ahmed (2007) establecen tres microfundamentos: (1) capacidad de adaptación (identificar y capitalizar oportunidades); (2) capacidad de absorción, basados en (Cohen \& Levinthal, 1990); (identificar, asimilar y explotar información de fuentes externas); (3) capacidad de innovación (desarrollo de nuevos productos y mercados). En un escrito de Pavlou \& El Sawy (2011), ellos proponen mantener la capacidad de aprendizaje en el sentido de (Teece \& Pisano, 1994), para incluirlo como un cuarto microcomponente. Wilhelm, Schlömer, \& Maurer (2015) establecen tres componentes, (1) detectar, como escanear el ambiente para identificar cambios y oportunidades; (2) aprendizaje, como el desarrollo de nuevas maneras de responder a los cambios y oportunidades; (3) reconfigurar, como la reorganización de rutinas. Aquí es necesario, recurrir a la experiencia obtenida en el desarrollo de la investigación, ya que tal como pudo observarse y como fruto de las entrevistas, el orden de los componentes de las capacidades dinámicas tal como lo propone Teece (2007), debe ser revaluado, es decir, "detectar" las oportunidades o las amenazas es un microcomponente fundamental, por cuanto, "una vez que la empresa ha incorporado esta capacidad a su inventario de actividades, lo que sigue es el componente de Reconfigurar o Transformar" (Teece, 2017, p. 4), la base de sus activos, para proceder a "Aprovechar" la oportunidad detectada o eludir la amenaza.

\subsection{La gestión de operaciones}

La gestión de operaciones como una función de las empresas manufactureras y de servicios, se ha convertido en una de las funciones cuyo abordaje se ha hecho desde la perspectiva de las capacidades dinámicas, Arafa \& EIMaraghy (2011) comentan que la capacidad dinámica de manufactura, en las organizaciones empresariales se conoce como flexibilidad de manufactura.

Haciendo una revisión de la literatura, encontramos a Buzacott \& Shanthikumar (1980), quienes establecieron que:

Un sistema de manufactura flexible (FMS) consiste en máquinas donde se realizan operaciones de producción, vinculadas por un sistema de manipulación de materiales y todas bajo control de un ordenador central. En contraste con una línea de transferencia donde todas las partes siguen la misma secuencia de operaciones, el sistema de manipulación de material permite que las partes sigan una variedad de rutas diferentes. El FMS debe ser capaz de procesar un gran volumen total de lotes de piezas pequeños a medianos (p. 339). 
Según Stecke \& Solberg (1981) el objetivo básico de la manufactura flexible es lograr la eficiencia, utilizando los niveles de la producción en masa. Al tiempo que mantiene la flexibilidad de los talleres de trabajo manuales. Las máquinas individuales deben ser bastante versátiles y capaces de realizar diferentes tipos de operación. Luego Browne, Dubois, Rathmill, Sethi, \& Stecke (1984), establecen ocho tipos de flexibilidades, las cuales denominaron, 1) Flexibilidad de máquina: facilidad de realizar los cambios necesarios para producir un conjunto de diferentes piezas. 2) Flexibilidad de proceso: capacidad de producir un conjunto dado de piezas, cada uno posiblemente utilizando diferentes materiales de varias maneras. 3) Flexibilidad de producto: capacidad de cambiar para producir un nuevo (conjunto de) producto (s) muy económica y rápidamente. 4) Flexibilidad de ruta: capacidad de manejar averías y continuar produciendo el conjunto dado de piezas. 5) Flexibilidad de volumen: capacidad de operar un FMS de manera rentable a diferentes volúmenes de producción. 6) Flexibilidad de expansión: capacidad de construir un sistema y expandirlo según sea necesario, fácil y modularmente. 7) Flexibilidad de operación: capacidad de intercambiar el orden de varias operaciones para cada tipo de pieza. 8) Flexibilidad de producción: el universo de tipos de piezas que el FMS puede producir.

Lim (1987) adiciona la flexibilidad de la mano de obra funcional, es decir, la adaptabilidad de los operarios a diferentes tareas y la flexibilidad de la mano de obra numérica, es decir, la capacidad de ajustar el número de operarios a las tareas de manufactura, posteriormente Sethi \& Sethi (1990), adicionan la flexibilidad de mercado, es decir, facilidad con la que el sistema de fabricación puede adaptarse a un entorno cambiante del mercado.

Handfield \& Pagell (1995), establecen que, la flexibilidad es un concepto multidimensional que apoya las respuestas organizacionales a la incertidumbre ambiental, permitiendo el logro de economías de alcance, donde la empresa con los mismos equipos puede manufacturar múltiples productos, con muy bajo costo, combinándolos. Para luego mostrar que la evolución de la flexibilidad se inicia con los equipos de control numérico y robots, cuyas aplicaciones incluyen soldadura por puntos, manejo de materiales, pintura en aerosol, inspección, corte, desbastado, envasado y sistemas de visión, continua con los sistemas de almacenamiento automatizado (AS), sistemas de recuperación (RS), diseño asistido por computador (CAD) y manufactura integrada por computador (CIM), esta última, establece vínculos en la cadena de valor, incluyendo diseño computarizado, planificación, manejo de materiales, fabricación y sistemas de apoyo (entrada de pedidos, contabilidad de costos, compras, etc.).

La flexibilidad, según Koste \& Malhotra (1999), está compuesta por diez dimensiones: maquina, mano de obra, manipulación de materiales, ruta, operación, expansión, volumen, mezcla, nuevo producto y modificación, cada una con sus propios elementos constitutivos, por lo que utilizan cuatro; alcance (número de opciones operaciones, tareas, productos, etc.), heterogeneidad (heterogeneidad de opciones - diferencias entre operaciones, tareas, productos, etc.), movilidad (penalización de transición-tiempo, costo, esfuerzo de transición) y uniformidad (similitud de los resultados de rendimiento-calidad, costos, tiempo, etc.), para entenderlas mejor.

El desarrollo y evolución del concepto de manufactura flexible, se ve complementado por otros conceptos que se generan en las plantas de producción, como también por parte de los estudiosos del tema productivo, así, en Japón, el sistema Toyota, se inicia al final de la segunda guerra mundial, aplicando los conceptos de control de calidad (QC), control total de calidad (TQC) y los métodos de la ingeniería industrial (IE), todos tomados de la industria norteamericana, cuya característica principal era la producción en masa. Sin embargo, Toyota requería un sistema, no para producir en masa, sino para producir en pequeños lotes y con varios diseños, para satisfacer ordenes de clientes. A este sistema Womack, Jones, \& Roos (1990), lo denomina Lean Manufacturing, por su énfasis en eliminar el desperdicio. 
El sistema Toyota, originado en el liderazgo de Ohno (1988), ha llegado a conocerse también como; justo a tiempo (JAT), cuya característica principal es la eliminación del desperdicio. Ohno, identifica siete tipos de desperdicios.

- $\quad$ Sobreproducción: Producir más o por adelantado.

- $\quad$ Filas: Tiempo ocioso, almacenamiento y espera.

- $\quad$ Transporte: Movimiento de materiales en más de una ocasión.

- Inventario: Materias primas innecesarias, trabajo en proceso, bienes terminados y exceso de suministros.

- Movimiento: Movimiento de equipo o personas.

- $\quad$ Sobreprocesamiento: Trabajo realizado sobre producto para repararlo.

- $\quad$ Producto defectuoso: Devoluciones, reclamos de garantía, repetición y sobrantes.

El modelo de producción en masa estadounidense fue desafiado en los años 80, cuando el modelo de producción esbelto de Japón proporcionó flexibilidad dentro de las líneas existentes, permitiendo el lanzamiento rápido, inducido por la demanda de nuevos productos y servicios. Debido a la confianza mutua de los socios del Keiretsu, el sistema de producción racional disminuyó tanto las incertidumbres del mercado como las de la tecnología y el peligro del oportunismo, lo que resultó en menores costos de transacción y, por consiguiente, un modo de fabricación menos jerárquico. La lógica de ganancias se basó principalmente en la explotación eficiente de la especialización y los esfuerzos innovadores de cada socio de la red (Kyläheiko \& Sandström, 2007).

La producción esbelta (Lean Manufacturing), es un enfoque multidimensional que abarca una amplia variedad de prácticas de gestión, incluyendo Justo a Tiempo (JIT), Gestión Total de la Calidad (TQM), Equipos de Trabajo (TW), Manufactura Celular, Gestión de Proveedores, Mantenimiento Preventivo Total (TPM) y Gestión de Recursos Humanos (HRM), en un sistema integrado. El núcleo principal de la manufactura es que estas prácticas pueden trabajar sinérgicamente para crear un sistema de alta calidad y racionalizado que manufactura productos terminados al ritmo de la demanda del cliente, con poco o ningún desperdicio (Shah \& Ward, 2003).

Retornando a los conceptos elaborados por Arafa \& ElMaraghy (2011), ellos establecen que las capacidades dinámicas están representadas en diez dimensiones de flexibilidad, máquina, manejo de materiales, operación, proceso, producto, ruta, volumen, expansión, programas de producción flexible y control, las cuales coinciden en mucho con las de Browne et al. (1984) y Koste \& Malhotra (1999). Sin embargo muchos autores adicionan prácticas como Six Sigma, planificación de recursos de la empresa (ERP) y muchos otros como parte de la flexibilidad de manufactura, lo cual por extensión se puede considerar como parte de las capacidades dinámicas que desarrolla la empresa para generar la ventaja competitiva que permite sostenerse en el tiempo.

Considerando este marco teórico, en la empresa objeto de estudio se realizó la investigación a fin de responder, ¿la flexibilidad de manufactura como Capacidad Dinámica, permite establecer que los microfundamentos son "Detectar, Aprovechar y Reconfigurar" o por el contrario solo son "Detectar y Reconfigurar"?

\section{Metodología}

Se realizó una investigación basada en métodos cualitativos; aquellos en donde Van Maanen (1979), afirma:

La etiqueta de los métodos cualitativos no tiene significado preciso en cualquiera de las ciencias sociales. Es en el mejor de los casos, un término general que abarca gran variedad de técnicas interpretativas que tratan de describir, decodificar, traducir, y de otra manera llegar a un acuerdo con el significado, no con la frecuencia de ciertos fenómenos que ocurren más o menos de forma natural en el mundo social (p. 520).

Dentro de los métodos cualitativos, se escogió el estudio de caso, dada la magnitud de la empresa y la variedad de elementos de la misma que se sometieron a estudio, de acuerdo con Yin (2003), un estudio de caso es una 
investigación empírica que investiga un fenómeno contemporáneo, en el contexto de la vida real, especialmente cuando los límites entre el fenómeno y el contexto no son claramente evidentes.

Esta investigación se llevó a cabo en la empresa FACAG, una de las principales productoras y comercializadoras de productos metálicos para la industria, el comercio y la construcción, ubicada en Colombia. Se hizo el análisis de los datos y las interpretaciones utilizando las herramientas propias de los métodos cualitativos, complementándolas con la triangulación; cuya operacionalización es como sigue; en primer lugar, se hicieron las entrevistas en profundidad, a 15 colaboradores de la empresa en cargos de presidente, vicepresidentes y directores de áreas, luego se cruzó la información obtenida mediante las entrevistas con los documentos de ventas, producción, logística, financiera, de calidad, ambiental y de riesgos, complementados con la observación directa en las plantas de operaciones.

El principal foco estuvo concentrado en la forma como se llevan a cabo las operaciones del día a día, ello fue la base para establecer la manera en que las actividades diarias daban como resultado, unos comportamientos que permiten la generación, codificación, difusión y aplicación de conocimientos relacionados con el flujo de operaciones que se realizan en la organización, lo cual permitió establecer la manera real en la cual los microfundamentos de las Capacidades Dinámicas apoyan los quehaceres en la organización, es decir, Detectar y Reconfigurar para poder Aprovechar.

Los microfundamentos detectar (es decir, monitorear el ambiente en busca de oportunidades o amenazas), aprovechar (es decir, beneficiarse de la oportunidad o eludir la amenaza) y reconfigurar (que consiste en adaptar los recursos en consonancia con las decisiones adoptadas), obedecen a una lógica secuencial que establece que solo se puede aprovechar cuando se han ejecutado las acciones con los recursos reconfigurados.

\section{Resultados}

La experiencia adquirida en el comercio de artículos de acero, permitió detectar la oportunidad de pasar a fabricante de los mismos y como consecuencia de ello, procede a reconfigurar su base de activos, es así como se inicia, todo el proceso de realizar los estudios necesarios para la puesta en funcionamiento de la planta transformadora de materias primas en artículos terminados para atender el mercado intermedio de constructores, ferreteros e industriales.

Desde su inicio, la planta contó con los más altos estándares de fabricación de productos de acero, la asesoría recibida de la empresa proveedora de la materia prima, marcó el nivel de eficiencia en la elaboración de los diferentes productos que debían fabricarse. Para atender la demanda la empresa implanta dos métodos de manufactura; fabricar por órdenes (make to order) y fabricar para inventarios (make to stock), estos dos sistemas permiten tener la necesaria flexibilidad para atender cualquier requerimiento de los clientes, lo mismo que mantener la planta en continuo funcionamiento, debido a que se trabaja con estimativos de venta.

Cuando se inició el proceso de manufactura, se mantenía la planta en funcionamiento las 24 horas, por orientación estrategica de la empresa, a fin de mantener productos en bodega para atender los requerimientos de los diferentes clientes. Esta orientación al producto se mantuvo durante mucho tiempo en la estrategia de la compañía, al fin de cuentas, el mercado protegido le permitía establecer los estimativos de la demanda y con base en esos estimativos se programaba la cantidad de inventario que debía mantenerse en bodegas para cumplir con la demanda requerida.

De esta orientación al producto se puede decir que permitió el arraigo y la habilidad para realizar las tareas con mucha destreza, ya que los procesos obedecían a cuidadosos planes de fabricación que poco a poco originaban los conocimientos necesarios para desempeñar con los más altos estándares de calidad las labores encomendadas. El metódico sistema de manufactura fue creando las condiciones necesarias para el surgimiento 
de una cultura de calidad en los procesos de fabricación que permitieron ganar ese espacio en la mentalidad de los clientes que permite gozar de la buena posición de mercado.

De los procesos seguidos en la planta se pueden describir dos de ellos que son el laminado en frio y el laminado en caliente. El laminado en caliente se le dice así porque el acero se deriva del mineral de hierro, el cual se convierte en planchas laminadas al rojo vivo, luego la pasan por bastidores y le disminuyen el espesor, es por eso que es en caliente, su nombre en inglés es Hot Rolled, enrollado en caliente; el laminado en frio se realiza en unos molinos, pero aplicando fuerza, no calor, y aun cuando se calienta, se le llama laminado en frío, el nombre en inglés es Cold Rolled; enrollado en frio, este último es para laminas delgadas.

En el año 1991, la planta contaba con una capacidad instalada para procesar 30.000 toneladas anuales, esta tarea la realizaban aproximadamente 100 empleados directos, en esa época la empresa no contrataba empleados temporales, para el año siguiente se inicia el proceso de globalización económica y por consiguiente se implementa la apertura económica, la cual fue vista por muchos empresarios como una gran amenaza, mientras los responsables de la empresa la vieron como una gran oportunidad, razón por la que desde entonces no ha dejado de crecer.

En la actualidad la capacidad instalada supera las 200.000 toneladas, contando con maquinaria de última generación, la cual le permite una gran flexibilidad en sus operaciones, precisamente por esa característica; la empresa segmenta su mercado objetivo en tres grandes focos: industria, grandes distribuidores y construcción. De esta manera se logra el alineamiento con la gran estrategia de colocar al cliente en el centro de las actividades de la empresa, buscando con ello adecuarse a la características del mercado, el cual había cambiado y la empresa no podía seguir haciendo lo mismo y esperar resultados diferentes.

Las operaciones cuentan con un sistema de planeación, que asume unas fechas de entrega y toda la programación queda sujeta a esos compromisos. El sistema de planeación se alimenta con la información generada por la fuerza de ventas, quienes visitan los clientes para establecer el cronograma de abastecimiento de acuerdo con las necesidades detectadas. Si surge una petición de algún producto especial, la fuerza de ventas lo comunica a la dirección de manufactura, la cual estudia la capacidad de sus equipos, si está en condiciones de producirlo, se lo comunica a mercadeo y se procede a tomar y fabricar el pedido.

Cuando la disponibilidad de maquinaria y equipo no es suficiente para atender la solicitud, se hace una primera aproximación por parte de la sección de proyectos, logística, mercadeo y manufactura, se analizan datos básicos sobre rentabilidad, si agrega valor al desempeño de la compañía, si está alineada con los objetivos y metas organizacionales. Se acude a la asesoría del área de mantenimiento, por lo de la adecuación y soporte de equipos, luego de lo cual, se procede a evaluar el proyecto en sus dimensiones básicas, esta información se envía a la alta gerencia, recomendando su ejecución.

En caso de no contar con los equipos y la tecnología adecuada, pero la demanda es prometedora y la sección de proyectos ha dado el aval para su realización, entonces se envía a la junta directiva, para que proceda en la consecución del capital para la puesta en marcha y autorice la continuidad de la operación. Entra luego el grupo de ingeniería a realizar un estudio de detalles, con el fin de iniciar todo el proceso, esto incluye un grupo interdisciplinario, para establecer el manejo ambiental, la parte de seguridad, aspectos básicos de producción, en fin que no quede algo por fuera, una vez se tiene toda esa información, se tasa en valores de mercado, se solicitan los equipos y maquinarias a los fabricantes, se realiza un cronograma de actividades incluyendo; montaje, puesta en marcha, capacitaciones del personal e inicio de operaciones.

En cuanto a la capacitación del personal, esto depende de la naturaleza del proyecto, si se tiene el personal, solo se le da el entrenamiento acorde con las nuevas especificaciones. Si es un proceso nuevo, se empieza desde antes y si se requiere, se envían a lugares donde deben capacitarlos, ya sea en los sitios de fabricación de la 
maquinaria o donde realizan procesos similares, de tal manera que puedan aprender. La capacitación se prolonga por el tiempo necesario, luego participan en el montaje y finalmente quedan en el equipo que opera el proceso.

Para efectos de contrarrestar la obsolescencia de algunos equipos o procesos, por alguna razón, ya sea por carencia de repuestos o haya que mejorar un proceso lo cual implica actualizar maquinaria, se procede a realizar un análisis, tratando de abarcar todas las posibles consecuencias, si la solución es sencilla y no requiere mayores inversiones, se realiza; si por el contrario es compleja y requiere inversiones, se procede como si se tratara de un proyecto, sin embargo, como este es de menor envergadura y además se cuenta con conocimiento se solicita solo la autorización.

La estrategia de colocar al cliente como centro, ha llevado a la compañía a ofrecer un servicio que facilita la fidelización. Cuando se presenta una requisición de algún cliente, la empresa envía ingenieros que se apersonan de las necesidades in situ, y conjuntamente estudian las alternativas de solución que amerita la problemática, eso se hace porque se cuenta con la infraestructura adecuada que permite responder a las necesidades detectadas en los clientes.

Para tener la flexibilidad necesaria, además de contar con la maquinaria adecuada, es indispensable contar con personal muy bien capacitado, multifuncional, es decir, que esté en capacidad de formar parte de cualquier equipo de operaciones en las diferentes secciones que conforman la planta. Una vez que la persona entra como empleado, se le capacita en las actividades del proceso por un lapso mínimo de dos meses, esto se hace en compañía de la persona que sea responsable de la actividad y por lo tanto que la conozca, de esta manera se transmite el conocimiento de un operario a otro.

En torno al proyecto de tubería, que se hizo para satisfacer las necesidades de los constructores de infraestructura, es decir aquellas compañías que se dedican a las obras civiles como puentes, carreteras, grandes superficies o centros comerciales y programas de viviendas, hubo necesidad de instalar toda la estructura, pues no se contaba con los equipos para la transformación de la materia prima en los productos requeridos por los constructores. El acero estructural que demanda el mercado obligó al montaje de toda una estructura nueva, tanto la tecnología como los conocimientos requeridos eran nuevos y aun se sigue aprendiendo, ya que todo lo que implica cambio genera contratiempos, los cuales son normales en este tipo de procesos.

En la actividad relacionada con la puesta en marcha de los equipos necesarios para la elaboración de la tubería, se partió de la fuerte cultura de la organización, la cual es abierta al cambio, las tecnologías blandas representadas en la gente que colabora facilitó la operación de esta nueva oferta de la empresa, es decir, la cultura de la organización se alineó para desplegar la estrategia, con lo cual se logra cambios en el ser, no es cuestión de equipos o maquinas, es la gente y su actitud lo que permite el buen funcionamiento de la planta.

La planta está aplicando el mantenimiento productivo total (TPM), el cual busca que esta produzca con altas eficiencias, con un altísimo nivel de excelencia operacional, lo cual implica contar con altos niveles de limpieza, altos niveles de seguridad, donde cada uno de los operarios es el gestor de su propio trabajo, es decir que cada operario se autogestiona, para ello se han capacitado alrededor de 300 personas en los principios que apoyan el TPM, o sea trabajos básicos, cómo elaborar estadísticas básicas, formas de desarrollar trabajo en planta, realización de mantenimiento, todo lo cual conduce al logro de un nivel muy alto de excelencia.

La materia prima importada se trae en unos pocos espesores, luego se somete a varios procesos y se establecen unas cuarenta referencias diferentes desde $1.9 \mathrm{~mm}$ hasta $0.27 \mathrm{~mm}$. A partir de estas referencias se pueden atender los pedidos generados por los clientes, de tal manera que la planta siempre está preparada para atender cualquier petición que se le haga, esa es la ventaja que se tiene cuando se trabaja en productos semiterminados, pues la planta cuenta con un proceso de premaquinado para que cuando se tenga el pedido del cliente, ya se cuenta con un proceso bastante adelantado. 
La planta está estructurada en forma de "V", es decir, inicia con pocas referencias de materias primas y se diversifica hacia diferentes procesos y productos, esto significa que la planta se abre y eso sucede porque las plantas en forma de "V", tienen mucha capacidad escondida, es decir, una vez que la materia prima se procesa, no hay manera de revertir el proceso, pues un producto formado no tiene forma de volverlo nuevamente a su forma inicial, por esa razón se mantiene una buena cantidad de productos semiterminados y con la ayuda de algoritmos desarrollados a partir de la metodología y la conceptualización de Teoría de Restricciones, se procede a elaborar la producción acorde con la demanda, es lo mismo que un sistema de jalonamiento, no de empujar, de tal manera que la demanda indica los procesos que deben iniciarse para cumplir con los requerimientos de los clientes.

La planta está diseñada para que los procesos vayan al ritmo de las necesidades de la demanda, esto se hace para no tener productos que alimenten el inventario, sino que vayan directamente a las bodegas de los clientes, para ello se apoya en la Teoría de Restricciones, Lean Manufacturing, Six Sigma y otras que perfeccionan el sistema para mantener las plantas bien ordenadas; se tienen productos a medio terminar que esperan la orden de continuar en algún proceso, obedeciendo a unas señales tipo semáforo, las cuales se activan cuando se dispone de órdenes de los clientes, así es como se conserva la agilidad y flexibilidad de la planta.

Al mantener productos semiterminados que se pueden utilizar en cualquiera de los procesos que se deben activar en fases subsiguientes para cumplir con la demanda de los clientes, la planta conserva su flexibilidad al tiempo que se ocupa en niveles de 85, 90 o 95\% de su capacidad, de esta manera cuando se tiene una petición de los clientes, se responde con mucha rapidez y prontitud, ya que la planta se mantiene con buenas agregaciones, que aun cuando son finitas, se cuenta con la posibilidad de tener adelantado el proceso en un gran porcentaje, lo que permite aprovechar la alta capacidad de la planta para procesar a una velocidad que es difícil de imitar por los competidores.

Los procesos que se utilizan en la planta, permiten mejor manejo de los inventarios, hasta el punto que se ha dado una reducción en los mismos, lo que significa el apego a los conceptos de (Lean Manufacturing), pues se trabaja con base en las necesidades reportadas por los clientes, ya que la idea es responder con prontitud, además al disminuir los inventarios, se facilita la visibilidad y también el manejo, por lo tanto aumenta la velocidad de rotación, lo que trae como consecuencia que se ocupe menos espacio en bodega, originando mejores flujos que impactan de manera positiva en los diferentes estados financieros que se utilizan para medir la capacidad de generar utilidades por parte de la organización.

\section{Conclusiones}

Como puede observarse, los procesos descritos dan cuenta de la forma como la organización ha desarrollado el conocimiento necesario para ejecutar sus actividades. Sus rutinas de trabajo, le han permitido perfeccionar las destrezas adecuadas para desempeñar las operaciones que se requieren para cumplir con los compromisos adquiridos por la fuerza de ventas con los clientes. Los factores que facilitan el excelente desempeño de la planta, se encuentran en la cuidadosa ejecución de las tareas, desde el mismo momento que inició la fabricación de los productos.

La planta siempre ha contado con los profesionales adecuados para desempeñar las labores necesarias. En la medida en que se ha requerido, se le ha dado la capacitación, con el fin de mantener a la fuerza de trabajo con los conocimientos y las habilidades que permitan ejecutar las actividades con la mayor solvencia posible, apuntando siempre a mantener los principios del Lean Manufacturing, para de esa forma generar el mayor beneficio, con miras a sostener la posición competitiva que se ha ganado en el mercado. 
Todo lo descrito, muestra de manera clara, que los microfundamentos en que se basan las Capacidades Dinámicas, se reafirman en la composición de estos, es decir, la empresa detecta oportunidades, realiza los estudios correspondientes y cuando las previsiones son positivas procede a reconfigurar su base de activos, lo cual le permite aprovechar los frutos por brindar una solución a las necesidades de los clientes.

\section{Referencias bibliográficas}

Arafa, A., \& ElMaraghy, W. (2011). Manufacturing strategy and enterprise dynamic capability. CIRP AnnalsManufacturing Technology, 60(1), 507-510.

Browne, J., Dubois, D., Rathmill, K., Sethi, S. P., \& Stecke, K. E. (1984). Classification of flexible manufacturing systems. The FMS magazine, 2(2), 114-117.

Buzacott, J. A., \& Shanthikumar, J. G. (1980). .Models for understanding flexible manufacturing systems. AlIE transactions, 12(4), 339-350.

Cohen , W., \& Levinthal, D. (1990). Absorptive capacity: a new perspective on learning and innovation. Administrative Science Quarterly, 35(1), 128-152.

Eisenhardt, K., \& Martin, J. (2000). Dynamic capabilities: What are they? Strategic Management Journal, 21(1), $1105-1121$.

Handfield, R. B., \& Pagell, M. D. (1995). An analysis of the diffusion of flexible manufacturing systems. International Journal of Production Economics, 39(3), 243-253.

Koste, L. L., \& Malhotra, M. K. (1999). A theoretical framework for analyzing the dimensions of manufacturing flexibility. Journal of Operations Management, 18(1), 75-93.

Kyläheiko, K., \& Sandström, J. (2007). Strategic options-based framework for management of dynamic capabilities in manufacturing firms. Journal of Manufacturing Technology Management,. (18, Trad.) 966984.

Lim, S. (1987). Flexible manufacturing systems and manufacturing flexibility in the United Kingdom. International Journal of Operations \& Production Management, 7(6), 44-54.

Ohno, T. (1988). Toyota production system: beyond large-scale production. New York: Productivity Press.

Pavlou, P. A., \& El Sawy, O. A. (2011). Understanding the elusive black box of dynamic capabilities. Decision sciences, 42(1), 239-273.

Sethi, A. K., \& Sethi, S. P. (1990). Flexibility in manufacturing: a survey. International journal of flexible manufacturing systems, 2(4), 289-328.

Shah, R., \& Ward, P. T. (2003). Lean manufacturing: context, practice bundles, and performance. Journal of Operations Management, 21(2), 129-149.

Stecke, K. E., \& Solberg, J. J. (1981). Loading and control policies for a flexible manufacturing system. The International Journal of Production Research, 19(5), 481-490.

Teece, D. (2003). Explicating dynamic capabilities: Asset selection, coordination, and entrepreneurship in strategic management theory. UC Berkeley.

Teece, D. J. (2007). Explicating dynamic capabilities: the nature and microfoundations of (sustainable) enterprise performance. Strategic management journal, 28(13), 1319-1350. 
Teece, D. J. (2017). Business models and dynamic capabilities. Long Range Planning, Article in press, 1-10.

Teece, D. J., Pisano, G., \& Shuen, A. (1997). Dynamic capabilities and strategic management. Strategic Management Journal, 18(7), 504-534.

Teece, D., \& Pisano, G. (1994). The dynamic capabilities of firms: an introduction. Industrial and corporate change, 3(3), 537-556.

Van Maanen, J. (1979). Reclaiming qualitative methods for organizational research: A preface. Administrative Science Quarterly, 520-526.

Wang, C., \& Ahmed, P. (2007). Dynamic capabilities: A review and research agenda. International Journal of Management Reviews, 9(1), 31-51.

Wilhelm, H., Schlömer, M., \& Maurer, I. (2015). How Dynamic Capabilities Affect the Effectiveness and Efficiency of Operating Routines under High and Low Levels of Environmental Dynamism. British Journal of management, 26(2), 327-345.

Womack, J. P., Jones, D. T., \& Roos, D. (1990). Machine that changed the world. New York: Simon and Schuster.

Yin, R. K. (2003). Case study research design and methods Applied social research methods series, 5 (third edition ed.).

Zollo, M., \& Winter, S. G. (1999). From organizational routines to dynamic capabilities. INSEAD, 38.

Esta obra está bajo una Licencia Creative Commons Attribución-NoCommercial 4.0 International

\section{(cc) EY-NC}

